Conduct problems, gender and adult psychiatric outcome of children with attention-deficit

\section{hyperactivity disorder}

\author{
SØREN DALSGAARD, PREBEN BO MORTENSEN, MORTEN FRYDENBERG \\ and PER HOVE THOMSEN
}

\author{
Background Attention-deficit \\ hyperactivity disorder (ADHD) is a \\ common childhood condition, and is more \\ prevalent in boys. The adult outcome of \\ girls with ADHD has never been studied.
}

\begin{abstract}
Aims To identify predictors for adult psychiatric outcome of children with

ADHD, including gender and comorbidity.
\end{abstract}

Method Children aged 4-15 years, referred for hyperactivity/inattention and treated with stimulants were included $(n=208)$. The Psychiatric Case Register provided follow-up data on psychiatric admissions in adulthood until a mean age of 31 years.

\section{Results A total of 47 cases (22.6\%) had a psychiatric admission in adulthood. Conduct problems in childhood were predictive (hazard ratio $\mathrm{HR}=2.3 ; 95 \%$ Cl I.22-4.33). Girls had a higher risk compared with boys $(H R=2.4 ; 95 \%$ Cl I.I-5.6).}

Conclusions Girls with ADHD had a higher risk of adult psychiatric admission than boys. Conduct problems were also associated with a higher risk. Girls with ADHD with conduct problems had a very high risk of a psychiatric admission in adulthood.

\section{Declaration of interest None.} Supported by The Danish Medical Council Research Foundation, Fru Hermansens Mindelegat and Rosalie Petersen Foundation.
Attention-deficit hyperactivity disorder (ADHD) is the one most commonly diagnosed psychiatric condition in childhood (American Psychiatric Association, 1994). The disorder is much more common in boys than in girls (Arnold, 1996) and comorbid disorders are frequent (Spencer et al, 1999). The prognosis of these children has been investigated extensively, regarding later academic performance, self-esteem and social functioning in adolescence and young adulthood, but not regarding adult mental status (Mannuzza \& Klein, 2000). The short-term follow-up studies of boys with ADHD have shown consistently that the most important predictors for poor adolescent outcome are comorbid conduct problems in childhood, low parental socioeconomic status and low IQ (Hechtman, 1999). No previous study has examined the adult outcomes of girls with ADHD.

\section{METHOD}

\section{Study population}

In a historical follow-up design, a cohort of referred children in the period 1969-1989 was identified. Cases were followed into adulthood in a nationwide register giving data on adult psychiatric status on all probands. Included in this study were all patients aged 4-15 years admitted to the Psychiatric Hospital for Children and Adolescents, Aarhus University Hospital in the period 1969-1989, who received pharmacological treatment with methylphenidate or dextroamphetamine. All probands were originally referred because of inattention and hyperactivity. A total of 218 probands were identified and case records for all of these were obtained. Using both DSM-IV and ICD-10 diagnostic criteria for ADHD, hyperkinetic disorder, oppositional defiant disorder (ODD), conduct disorder and other comorbid disorders, case records were reassessed diagnostically. Probands were divided into three ADHD groups: definite ADHD; sub-threshold ADHD; and possible ADHD. Definite ADHD included probands fulfilling the specified DSM-IV criteria. Sub-threshold ADHD included probands lacking one or two criteria for ADHD under the DSM-IV. Possible ADHD included the rest of the probands, who lacked more than two criteria in meeting the full ADHD. Rather than excluding probands not meeting criteria for definite ADHD, all probands were included in the follow-up and the psychiatric outcome of these three groups was compared. The exclusion disorders were pervasive developmental disorder (PDD; DSM 299 or ICD10 F84) and psychosis (DSM 293, 295, 297-298 or ICD-10 F20-29). Because of PDD, 10 children were excluded, leaving 208 probands (183 boys and 25 girls) included in the study. A detailed description of the method has been published previously (Dalsgaard et al, 2001). The IQ, prevalence of comorbid disorders, socio-economic status, Children's Global Assessment Scale (CGAS; Shaffer et al, 1983) and aspects of the pharmacological treatment with stimulants were also reassessed retrospectively from case records, as shown in Table 1.

\section{Reliability}

Reassessment of 191 case records for ADHD or hyperkinetic disorder and ODD or conduct disorder was performed blindly by two independent raters: a resident child and adolescent psychiatrist (rater 1); and an experienced senior child psychiatrist (rater 2). The first 23 case records were included in a consensus rating. After the consensus rating, the two raters did not discuss their ratings of the remaining 168 cases. This reliability study of ADHD diagnoses from 168 records showed a satisfactory agreement of $76 \%$ with a corresponding Cohen's kappa of 0.51 . The agreement on hyperkinetic disorder was slightly better, at $77 \%$ and a kappa of 0.54 . In diagnosing conduct disorder and ODD, higher agreement percentages were found, in the range $73-92 \%$ and kappas in the range $0.44-0.70$. There were no gender differences in the reliability of the diagnosis of ADHD in this reassessment. There was no systematic difference in the rating of any of the specific ADHD or hyperkinetic disorder items nor was there any significant difference in associations between correlated items between the two 
Table I Baseline description of sample, at initial inclusion $(n=208)$

\begin{tabular}{|c|c|c|}
\hline \multicolumn{3}{|l|}{ Gender ( $n(\%))$} \\
\hline Male & \multicolumn{2}{|c|}{$183(88)$} \\
\hline Female & 25 & $(12)$ \\
\hline $\begin{array}{l}\text { Age at initial inclusion, years } \\
\text { (mean (s.d.)) }\end{array}$ & 8.0 & $(2.2)$ \\
\hline \multicolumn{3}{|c|}{ Attention-deficit hyperactivity disorder ( $(\%))$} \\
\hline Definite diagnosis & 135 & (64.9) \\
\hline Sub-threshold diagnosis & 32 & $(15.4)$ \\
\hline Possible diagnosis & 41 & $(19.7)$ \\
\hline \multicolumn{3}{|l|}{ IQ (n (\%)) } \\
\hline Average $(\mathrm{IQ}>70)$ & 176 & $(84.6)$ \\
\hline $\begin{array}{l}\text { Mild ICD mental retardation } \\
\qquad(\text { IQ 50-70) }\end{array}$ & 27 & $(13.0)$ \\
\hline $\begin{array}{l}\text { Moderate ICD mental } \\
\text { retardation (IQ 35-49) }\end{array}$ & 5 & (2.4) \\
\hline \multicolumn{3}{|c|}{ WPPSI, WISC or WISC-R' (mean (s.d.)) } \\
\hline Verbal IQ & 84.2 & $(18.7)$ \\
\hline Performance IQ & 85.5 & $(19.8)$ \\
\hline Total IQ & 84.1 & $(17.5)$ \\
\hline \multicolumn{3}{|c|}{ Prevalence of comorbidity in childhood $(n(\%))$} \\
\hline Oppositional defiant disorder & 77 & $(37.0)$ \\
\hline Conduct disorder & 34 & $(16.3)$ \\
\hline $\begin{array}{l}\text { Oppositional defiant disorder or } \\
\text { conduct disorder }\end{array}$ & 88 & $(42.3)$ \\
\hline Learning disorders & 126 & $(60.6)$ \\
\hline Motor skills disorder & 116 & (55.8) \\
\hline Anxiety disorders & 52 & $(25.0)$ \\
\hline Tic disorders & 19 & $(9.1)$ \\
\hline $\begin{array}{l}\text { Obsessive-compulsive } \\
\text { disorders }\end{array}$ & 4 & (1.9) \\
\hline \multicolumn{3}{|l|}{ Parental socio-economic status (n (\%)) } \\
\hline High (I, II) & 16 & (7.7) \\
\hline Middle (III, IV) & 85 & (40.9) \\
\hline Low (V) & 107 & $(51.4)$ \\
\hline \multicolumn{3}{|l|}{ Referral source $(n(\%))$} \\
\hline Educational psychologist & 71 & $(34.1)$ \\
\hline General practitioner & 34 & $(16.3)$ \\
\hline Social services & 13 & $(6.3)$ \\
\hline Other & 90 & (43.3) \\
\hline
\end{tabular}

Terms of admission at initial inclusion ( $n(\%))$

$\begin{array}{lrr}\text { In-patient } & 191 & (91.8) \\ \text { Out-patient } & 17 & (8.2)\end{array}$

Children's Global Assessment Scale (n (\%))

$\begin{array}{lrr}\text { Only slightly affected (70-100) } & 4 & (1.9) \\ \text { Moderately affected (50-60) } & 78 & (37.5) \\ \text { Severely affected (below 50) } & 126 & (60.6)\end{array}$

WISC, Wechsler Intelligence Scale for Children; WPPSI, Wechsler Preschool and Primary Scale of Intelligence. I. $n=|5|$. raters. Rater 1 reassessed the complete sample of 208 cases and this rating was used as the final diagnostic conclusion.

\section{Assessment of adult psychiatric admissions}

Data from the Danish Psychiatric Central Register (DPCR; Munk-Jorgensen \& Mortensen, 1997) gave complete followup information on all probands regarding any in-patient and day patient psychiatric admissions. DPCR is a nationwide computerised register containing data on all admissions to Danish psychiatric in-patient facilities since April 1969 and also including out-patient consultations at psychiatric departments since 1995. The register contains data on dates of admissions, and discharges, terms of admissions, ICD-8 diagnoses (World Health Organization, 1967) for the period 1969-1993 and ICD-10 diagnoses (World Health Organization, 1992) from January 1994. Because all included patients had at least one previous psychiatric admission, the index follow-up event was defined as the first in-patient admission to a psychiatric hospital ward after the age of 15 years. Data were censored on either the date of the first psychiatric admission after the age of 15 , date of death or 15 June 2000 , whichever came first. In terms of lifetime diagnosis of four disorders - schizophrenia, personality disorder, mood disorder and substance use disorder - during the 10- to 38-year follow-up period data were censored using the same criteria. Cases with a lifetime diagnosis of one of these four diagnoses were censored on the date of their first adult psychiatric admission and those without a lifetime diagnosis of any of these four disorders but with other disorders, were censored on the date of their first other adult psychiatric admission.

\section{Diagnostic groups}

The follow-up register data included both ICD-8 and ICD-10 diagnoses. Eight different diagnostic groups were formed using the guidelines provided by the World Health Organization (1999): schizophrenia and schizophreniform psychosis (295.09295.99, 297.09-297.99, 298.39 and F20 29); affective psychosis (296.09-296.99, 298.09, 298.19 and F30-39); antisocial personality disorder $(301.79,301.39$, 301.82, 301.83 and F60.2); other personality disorders (301 and F60-69 except F60.2); mental retardation (310-315 and
F70-79); abuse of alcohol (291, 303 and F10.x); substance misuse disorder (304 and F11-19); and finally the last group, including all other diagnoses.

\section{Statistical analysis}

Follow-up data were analysed using survival analyses. Kaplan-Meier survival plots were used in univariate analyses, and to test equality in survival distributions for gender and conduct problems log rank tests were applied. Hazard ratios (HRs) by Cox regression were used in the predictor analyses, giving crude HRs with 95\% confidence intervals for baseline variables. In the multivariate analyses odds ratios were adjusted for gender and conduct problems. Possible interactions between gender and conduct problems and other potential long-term predictors were tested using Wald tests. The SPSS 9.0 (SPSS Inc., 1998) and STATA 6.0 (StataCorp, 1999) statistical packages were used in the analyses.

\section{RESULTS}

\section{Adult psychiatric diagnoses}

At the end of this 10- to 30-year follow-up study the 208 cases had a mean age of 31 years. Of the 208 included cases of ADHD, $47(22.6 \%)$ experienced an adult psychiatric admission in the follow-up period at a mean age of 23 years at the time of their first adult psychiatric admission. According to data from the Danish causes of death register (Juel \& Helweg-Larsen, 1999) two probands died in the follow-up period, and both were male. One of these had five psychiatric admissions in adulthood under diagnoses of antisocial personality disorder and misuse of alcohol before he died aged 25 years by suicide from a prescriptiondrug overdose. The other male died aged 27 years from an accidental overdose of morphine. The number of adult psychiatric admissions among the 47 cases varied substantially, ranging from 1 to 36 with a median of 2 admissions.

The most prevalent diagnosis at the first adult psychiatric admission was any personality disorder (accounting for $29 \%$ of the cases), half of which were antisocial personality disorder. The second most prevalent single diagnosis at first adult psychiatric admission was mood disorder. Personality disorder was also the most prevalent lifetime diagnosis in this followup until a mean age of 31 years. Although 
Table 2 In-patient psychiatric admissions in adulthood, at follow-up' $(n=208)$

\begin{tabular}{|c|c|c|}
\hline Psychiatric follow-up data on number of probands ( $n(\%))$ & 208 & $(100)$ \\
\hline Number of deaths among probands at time of follow-up ( $n(\%))$ & 2 & (I) \\
\hline Probands with a psychiatric admission in adulthood $(n(\%))$ & 47 & $(22.6)$ \\
\hline Age at follow-up (mean (s.d.)) & 31.1 & $(6.6)$ \\
\hline Age at first adult psychiatric admission (mean (s.d.)) & 23.2 & $(5.5)$ \\
\hline \multicolumn{3}{|l|}{ ICD-8/I0 diagnosis at first adult psychiatric admission ( $n(\%))$} \\
\hline Any personality disorder & 14 & (6.8) \\
\hline Antisocial personality disorder & 7 & (3.4) \\
\hline Mood disorder & 7 & (3.4) \\
\hline Mental retardation & 2 & $(1.0)$ \\
\hline Adjustment disorder & 2 & (1.0) \\
\hline Substance or alcohol use disorder & 6 & (1.4) \\
\hline Schizophrenia & 2 & $(1.0)$ \\
\hline Mental observation & 3 & (1.4) \\
\hline Other & II & (5.3) \\
\hline \multicolumn{3}{|l|}{ Lifetime diagnoses,' ICD-8/I0 ( $(\%))$} \\
\hline Any personality disorder & 18 & (8.7) \\
\hline Antisocial personality disorder & 10 & $(4.8)$ \\
\hline Schizophrenia & 8 & (3.8) \\
\hline Mood disorder & 8 & (3.8) \\
\hline Substance or alcohol use disorder & 15 & (7.2) \\
\hline
\end{tabular}

I. Follow-up was until a mean age of 31 years.

only two cases were given a diagnosis of schizophrenia at the first adult psychiatric admission, the lifetime prevalence for schizophrenia was much higher $(3.8 \%)$, as shown in Table 2 . The lifetime prevalence of antisocial personality disorder was $4.8 \%$.

\section{Long-term predictors}

Gender was the single most important predictor for a psychiatric admission in adulthood. Thirty-two per cent of the female probands had a psychiatric admission in adulthood. Being female was associated with a significantly higher risk for a later admission (HR $=2.42$; 95\% CI 1.05-5.62). A comorbid diagnosis of either ODD or conduct disorder in childhood was also predictive ( $\mathrm{HR}=2.29$; 95\% CI 1.22-4.33). Most other comorbid disorders in childhood, including learning and communication disorders and motor skills disorder, lowered the risk of later psychiatric admissions, although not in a statistically significant manner. A high total IQ was associated with a small but statistically significantly higher risk. When adjusted for gender and conduct problems the tendency was not significant. The adult psychiatric outcome of children with definite ADHD was not different from the outcome of children with sub-threshold or possible ADHD. The number of inattentive, hyperactive or impulsive symptoms in childhood was not predictive nor was the socio-economic status of the parents. A severe global impairment in childhood, measured as a CGAS score below 50, did not increase the risk for an adult psychiatric admission when adjusted for gender and conduct problems. Among probands without conduct problems, $12 \%$ experienced a psychiatric admission in the follow-up compared with $27 \%$ of the probands with conduct problems $(P=0.017)$. The overall results of the predictor analyses are shown in Table 3. Duration of treatment with stimulants in childhood was not associated significantly with the adult psychiatric outcome, and did not predict later psychotic episodes or substance misuse disorder.

\section{Gender and conduct problems}

Girls with ADHD and comorbid conduct problems had a much higher risk of an adult psychiatric admission compared with those without conduct problems $(\mathrm{HR}=6.0$; $95 \%$ CI 1.21-30.09). Of the 25 female probands, 10 had had comorbid conduct problems in childhood. Sixty per cent of these females with ADHD and comorbid conduct problems experienced an adult psychiatric admission. The survival analysis according to both gender and the presence of ODD or conduct disorder are shown in Fig. 1. Independent of gender, the severity of the conduct problems also showed a dose-response relationship with adult psychiatric outcome. Mild conduct problems tended to predict a higher risk; however, moderate or severe conduct problems predicted a much higher risk and the poorest adult psychiatric outcome $(\mathrm{HR}=3.10$; 95\% CI 1.35-7.16) compared with having no conduct problems. The three most important ODD or conduct disorder symptoms in predicting poor adult psychiatric outcome seem to be: 'has deliberately destroyed others' property (other than fire setting)' ( $O R=2.6 ; 95 \%$ CI 1.1-6.2); 'is often truant from school, beginning before age 13 years' $(\mathrm{OR}=2.5$; $95 \%$ CI $1.1-5.5)$; and 'often initiates physical fights' $(\mathrm{OR}=2.0 ; 95 \%$ CI 1.1-3.6). The last of these was a frequent comorbid symptom, present in more than half the cases, whereas the first two symptoms were less common. In a test for any possible interactions, tic disorder and gender was the only significant interaction identified. Girls with comorbid tic disorder had a significantly higher risk compared with boys with comorbid tics. Other potential long-term predictors were also tested but no significant interactions were identified.

\section{Predictors for lifetime diagnoses}

The 10 cases with a later diagnosis of antisocial personality disorder all had some conduct problems in childhood and a diagnosis of conduct disorder predicted antisocial personality disorder $(\mathrm{HR}=3.78$; 95\% CI 1.06-13.44). Antisocial personality disorder was not predicted by gender, IQ, duration of stimulant treatment, or the degree of inattention, hyperactivity or impulsivity in childhood. Being female predicted a lifetime diagnosis of schizophrenia (HR=6.56; 95\% CI 1.39-30.94). A comorbid diagnosis of ODD or conduct disorder in childhood also tended to increase the risk, although not statistically significantly when adjusted for gender. Comorbid anxiety in childhood was not associated with later schizophrenia. Having mild or moderate global impairment in childhood, with a CGAS above 50, tended to increase the risk for a lifetime diagnosis 
Table 3 Long-term childhood predictors for an adult psychiatric admission. Crude and adjusted hazard ratios (HRs) and $95 \%$ confidence intervals

\begin{tabular}{|c|c|c|}
\hline Variable & Crude HR $(95 \% \mathrm{Cl})$ & $\begin{array}{c}\text { Adjusted HR' } \\
(95 \% \mathrm{Cl})\end{array}$ \\
\hline Gender, girls v. boys & $1.62(0.76-3.48)$ & $2.42(1.05-5.62)$ \\
\hline Diagnosis of ODD or conduct disorder & $1.86(1.05-3.31)$ & $2.29(1.22-4.33)$ \\
\hline \multicolumn{3}{|l|}{ ADHD subtypes } \\
\hline Hyperactive impulsive type & $1.20(0.47-3.13)$ & $\mathrm{I} .15(0.44-2.96)$ \\
\hline Combined type & $1.14(0.50-2.60)$ & $1.04(0.45-2.38)$ \\
\hline Inattentive type & 1.00 & 1.00 \\
\hline Learning disorder & $0.75(0.42-1.34)$ & $0.74(0.42-1.33)$ \\
\hline Communication disorder & $0.90(0.51-1.6 I)$ & $0.99(0.55-1.76)$ \\
\hline Motor skills disorder & $0.79(0.45-1.4 I)$ & $0.79(0.44-I .4 I)$ \\
\hline Anxiety disorder & $1.05(0.54-2.07)$ & $0.99(0.50-1.96)$ \\
\hline Tic disorder & $0.72(0.29-I .83)$ & $\mathrm{I} .44(0.56-3.67)$ \\
\hline Deficits in attention, motor control and perception & $0.92(0.46-1.85)$ & $0.91(0.45-1.84)$ \\
\hline Severely impaired at inclusion (CGAS $<50$ v. CGAS $>50$ ) & $1.27(0.70-2.29)$ & $0.99(0.5 I-1.95)$ \\
\hline Low parental SES $v$. high or medium, at inclusion & $1.66(0.92-2.99)$ & $1.67(0.92-3.04)$ \\
\hline Low IQ $(<70) v$. high or medium IQ $(\geqslant 70)$ & I.I5 (0.54-2.46) & $1.39(0.65-3.00)$ \\
\hline \multicolumn{3}{|l|}{ IQ at inclusion, WPPSI, WISC or WISC- $\mathrm{R}^{2}$} \\
\hline Verbal IQ & I.I7 (0.98-I.38) & $1.13(0.95-1.36)$ \\
\hline Performance IQ & I.II (0.94-I.32) & $\mathrm{I} .07(0.90-\mathrm{I} .27)$ \\
\hline Total IQ & $1.22(1.01-1.47)$ & I. $17(0.97-1.42)$ \\
\hline
\end{tabular}

ADHD, attention-deficit hyperactivity disorder; CGAS, Children's Global Assessment Scale; ODD, oppositional defian disorder; SES, socio-economic status; WISC, Wechsler Intelligence Scale for Children; WPPSI, Wechsler Preschool and Primary Scale of Intelligence.

I. Adjusted for gender and diagnosis of ODD or conduct disorder.

2. $n=150$, HRs per $10 \mathrm{IQ}$ points. of schizophrenia, compared with having severe impairment. There was a higher risk for a lifetime diagnosis of a mood disorder among girls $(\mathrm{HR}=5.30 ; 95 \% \mathrm{CI}$
1.24-22.67). Mood disorder was not predicted by anxiety in childhood. Substance misuse disorder was associated with being female $(\mathrm{HR}=18.33 ; 95 \%$ CI

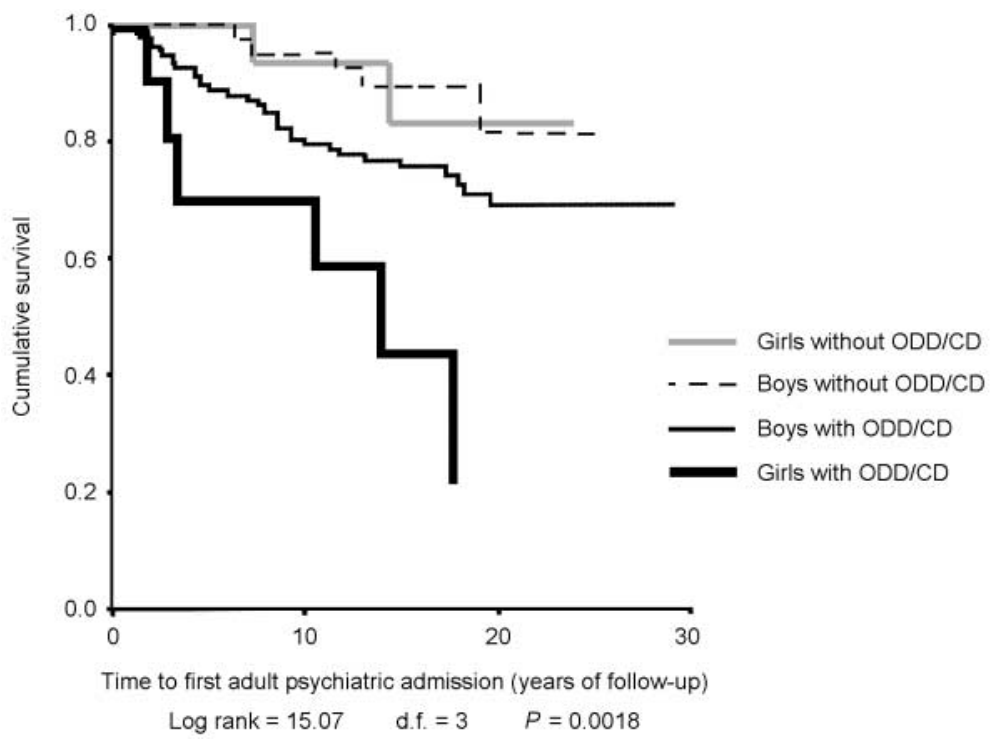

Fig. I Kaplan-Meier estimates of the time of follow-up from 15 years of age to the first psychiatric admission, stratified by gender and presence of symptoms of oppositional defiant disorder (ODD) or conduct disorder (CD) in childhood.
3.17-105.96) and also predicted by ODD or conduct disorder $(\mathrm{HR}=23.69 ; 95 \% \mathrm{CI}$ 3.40-165.39).

\section{DISCUSSION}

\section{Limitations and strengths of the present study}

Despite the fact that the prognosis for children with ADHD has been investigated extensively, no previous study has examined the long-term prognosis of girls with ADHD. To our knowledge the present study also constitutes the longest and the largest follow-up study of children with ADHD so far. In addition to the sample size and the length of follow-up, using register data gives a complete follow-up on all cases without the attrition that in many followup studies is often a limitation, especially on psychiatric populations. This is also the first study to evaluate whether comorbid conduct problems in children with ADHD predict psychiatric admissions in adulthood.

The present study does have limitations. First, the cases included were all referred to the same child and adolescent psychiatric clinic and were all treated with stimulants. Accordingly, our results might not be generalisable to community-based samples of children with ADHD. Second, in this historical follow-up study, the more specific characteristics of the cases in childhood were reassessed from case records, based upon secondary data. However, an interrater-reliability study was performed showing good quality of these data (Dalsgaard et al, 2001). Finally, as the study also aims to evaluate the long-term outcome of girls with ADHD and the predictive value of gender, it is a limitation to have included only 25 girls.

\section{Psychiatric status in previous follow-up studies of children with ADHD}

Numerous follow-up studies have reported on the adolescent outcome of children with ADHD, especially boys. Only three studies have focused on the adult mental status. However, no studies have identified convincing, sturdy predictors of adult mental outcome.

The Montreal Study conducted a 15year follow-up study of 104 clinically referred probands (Weiss et al, 1985). Only $49-61 \%$ of the initial sample completed the follow-up at age 25 years. No specific 
variable predicted psychiatric outcome; however, low IQ and socio-economic status, emotional instability, aggressiveness and poor parental mental health predicted a poor global outcome (Hechtman et al, 1984). The New York studies were performed on clinical samples of boys with hyperactivity without any conduct problems, with follow-up at age 24 and 26 years (Mannuzza et al, 1993, 1998). Neither IQ nor parental psychopathology was predictive of adult outcome. Because children with comorbid conduct problems were excluded from these studies, the findings suggest that even children without conduct problems have a higher risk of antisocial personality disorder in adulthood compared with controls. A small subsample of hyperactive girls $(n=12)$ were followed into adolescence (Mannuzza \& Gittelman, 1984), and compared with male controls. The girls with ADHD had poorer outcome in terms of social, academic and behavioural functioning in adolescence. Unpublished data indicated that females in adulthood had better outcome than males, with lower risk of antisocial personality disorder and substance misuse disorder (Mannuzza \& Klein, 2000). The Göteborg Community-Based Study (Rasmussen \& Gillberg, 2000) was a long-term follow-up of 55 cases at age 22 years. A prediction analysis was not published but the poorest outcome was actually seen in cases with developmental coordination disorder only, without ADHD. The Loney Study found low IQ in childhood to be the strongest predictor of antisocial personality disorder and alcoholism (Loney et al, 1983). Aggression in childhood was associated with later weapon use, police contacts and the use of hallucinogens, but not with adult mental status.

\section{The present study compared with previous follow-up studies}

The prevalence of any psychiatric disorder in adulthood was slightly lower in our study $(23 \%)$ compared with previous studies $(33-52 \%)$. This could be explained by the fact that only psychiatric admissions to hospitals in adulthood were used as a measure of outcome in the present study. Probands might have developed psychiatric disorders in adulthood without being admitted and the prevalence could thereby be underestimated. This could also explain why the prevalence of antisocial personality disorder was lower than in previous studies.
Personality disorders and substance/alcohol misuse disorder being the two most prevalent diagnoses at adult follow-up is very much consistent with previous findings. The low prevalence rates of mood disorders were also comparable. Both present and lifetime diagnoses of schizophrenia were elevated in the present study compared with the previous studies; this could be explained by the longer follow-up and the complete follow-up on all probands in our study. Patients with psychosis are more likely to have been missed because of attrition in previous long-term follow-up studies.

\section{Comparison of predictors}

No solid childhood characteristics have previously been found to predict adult psychiatric outcome. IQ and parental socioeconomic status have previously been found to be associated negatively with a poorer global adult outcome. Conduct problems in childhood have previously been found to predict antisocial behaviour in adolescence but the previous longer follow-up studies have not been able to conclude on the predictive value of such comorbidity. The findings from the present study on the importance of conduct problems in childhood in predicting adult psychiatric outcome is supported by the short-term follow-up studies. In differentiating between conduct problems, not only the aggressive symptoms seem to increase the risk of adult psychiatric admissions.

When adjusting for gender and presence of conduct problems in childhood parental socio-economic status did not predict poor psychiatric outcome. The severity of inattentiveness, hyperactivity and impulsivity did not predict adult psychiatric status, nor did global impairment measured with CGAS when adjusted for gender and conduct problems. Duration of stimulant treatment was not randomised in the present study and the association between long duration and later adult psychiatric admissions is most likely to be caused by higher severity or more impairment of the cases treated longer (Hechtman et al, 1981). Regarding IQ, the present study found a very small but significant association but the direction of this was opposite to previous findings, as a higher IQ predicted a poorer outcome (crude $\mathrm{HR}=1.22$; 95\% CI 1.01-1.47 per 10 IQ points). Indeed, IQ was not a strong predictor in the present study. In the interpretation of the results it is important to emphasise that the predictive value of a large number of variables was tested in the present study. In the systematic analyses of interactions, the small sample size in the different groups gives very little data and limited information on interactions.

\section{Gender as a predictor}

Girls with ADHD often differ from boys in terms of lower levels of hyperactivity and lower rates of externalising behaviours. Although boys more often have comorbid conduct problems, girls with ADHD and ODD or conduct disorder might have more social problems than boys (Carlson et al, 1997; Gaub \& Carlson, 1997; Faraone et al, 2000). It has also been suggested that girls with ADHD are less vulnerable to deficits in executive functions than boys with ADHD (Seidman et al, 1997). A recently published brain imaging study suggests that some of the morphological differences seen in boys with ADHD are also seen in girls with ADHD (Castellanos et al, 2001). Overall, the research on gender differences in ADHD have not established any good biological differences, although girls with ADHD have different neuropsychological profiles, patterns of comorbidity, severity of core symptoms and impairment in social functioning compared with boys with ADHD.

Our study suggests that girls with ADHD might have a poorer adult psychiatric outcome than boys with ADHD. However, the gender differences in risk of a psychiatric admission in adulthood found in the present study might only reflect a high risk for women in general. The finding could also be explained by a true biological gender difference in the disorder or a different threshold for referral for girls. Although girls seldom exhibit comorbid conduct problems, such comorbidity in girls seems to be far more important in predicting adult psychiatric outcome than IQ, parental socioeconomic status, global impairment and severity of core symptoms of ADHD. More long-term studies on larger samples of girls with ADHD, are needed to confirm this.

\section{ACKNOWLEDGEMENTS}

P.B.M. is in part financed by the Theodore and Vada Stanley Foundation. The National Centre for Register-based Research is funded by The Danish Research Foundation. We thank Niels Hansen, MD, Senior Consultant Child Psychiatrist for his assistance as clinical rater in the reliability study and 
Merete Juul Sørensen, Frank Verhulst and Niels Bilenberg for encouraging and helpful comments on early ideas for this paper.

\section{REFERENCES}

American Psychiatric Association (1994) Diagnostic and Statistical Manual of Mental Disorders (4th edn) (DSM-IV). Washington, DC: APA

Arnold, L. E. (1996) Sex differences in ADHD: Conference summary. Journal of Abnormal Child Psychology, 24, 555-569.

Carlson, C. L., Tamm, L. \& Gaub, M. (1997) Gender differences in children with ADHD, ODD, and co-occurring ADHD/ODD identified in a schoo population. Journal of the American Academy of Child and Adolescent Psychiatry, 36, 1706-1714.

Castellanos, F. X., Giedd, J. N., Berquin, P. C., et al (200I) Quantitative brain magnetic resonance imaging in girls with attention-deficit/hyperactivity disorder. Archives of General Psychiatry, 58, 289-295.

Dalsgaard, S., Hansen, N., Mortensen, P. B., et a (200I) Reassessment of ADHD in a historical cohort of children treated with stimulants in the period 1969-1989. European Child and Adolescent Psychiatry 10, 230-239.

Faraone, S.V., Biederman, J. \& Monuteaux, M. C. (2000) Attention-deficit disorder and conduct disorder in girls: evidence for a familial subtype [in process citation]. Biological Psychiatry, 48, 21-29.

Gaub, M. \& Carlson, C. L. (1997) Gender differences in ADHD: a meta-analysis and critical review. Journal of the American Academy of Child and Adolescent Psychiatry 36, 1036-1045.

Hechtman, L. (1999) Predictors of long-term outcome in children with attention-deficit/hyperactivity disorder. Pediatric Clinics of North America, 46, 1039-1052.

_ , Weiss, G., Perlman, T., et al (1981) Hyperactives as young adults: prospective ten-year follow-up. In Psychosocial Aspects of Drug Treatment for Hyperactivity (eds K. D. Gadow \& J. Loney), pp. 417-442. Boulder CO: Westview Press.

, et al (1984) Hyperactives as young adults: initial predictors of adult outcome. Journal of the American Academy of Child Psychiatry, 23, 250-260.

Juel, K. \& Helweg-Larsen, K. (1999) The Danish registers of causes of death. Danish Medical Bulletin, 46 , 354-357.

Loney, J., Whaley-Klahn, M. A., Kosier, T., et al (1983) Hyperactive boys and their brothers at 2l: predictors of aggressive and antisocial outcomes. In Prospective Studies of Crime and Delinquency (eds K. T. Van Dusen \& S. A. Mednick), pp. I8I. Boston, MA: Kluwer-Nijhoff.

Mannuzza, S. \& Gittelman, R. (1984) The adolescent outcome of hyperactive girls. Psychiatry Research, $\mathbf{1 3}$ 19-29.

— \& Klein, R. G. (2000) Long-term prognosis in attention-deficit/hyperactivity disorder. Child and Adolescent Psychiatric Clinics of North America, 9 7II-726.

_ , _ , Bessler, A., et al (1993) Adult outcome of hyperactive boys. Educational achievement, occupational rank, and psychiatric status. Archives of General Psychiatry, 50, 565-576.

\section{CLINICAL IMPLICATIONS}

- Girls with attention-deficit hyperactivity disorder (ADHD) had a higher risk of psychiatric admissions in adulthood compared with boys with ADHD.

- Comorbid conduct problems were, independent of gender, associated with a higher risk.

- Although girls with ADHD compared with boys with ADHD seldom have conduct problems, such comorbidity is important for the prognosis.

\section{LIMITATIONS}

- As all probands were referred cases and were all treated with stimulants, the results may not be generalisable to all children with ADHD.

- The specific characteristics of the cases in childhood were reassessed from case records, based upon secondary data but an interrater-reliability study was performed showing good quality of these data.

- The study aims to evaluate the long-term outcome of girls with ADHD but is limited by only having included 25 girls.

SØREN DALSGAARD, MD, PhD, Psychiatric Hospital for Children and Adolescents, Aarhus University Hospital, Denmark; PREBEN BO MORTENSEN, DMed Sci, National Centre for Register-Based Research, Aarhus University, Denmark; MORTEN FRYDENBERG, PhD, Department of Biostatistics, Aarhus University, Denmark; PER HOVE THOMSEN, DMed Sci, Psychiatric Hospital for Children and Adolescents, Aarhus University Hospital, Denmark

Correspondence: Søren Dalsgaard, Psychiatric Hospital for Children and Adolescents, Harald Selmersvej 66, 8240 Risskov, Aarhus University Hospital, Denmark. E-mail: sda@buh.aaa.dk

(First received 2 January 2002, final revision 8 May 2002, accepted 29 May 2002)

$\ldots, \ldots$, , et al (1998) Adult psychiatric status of hyperactive boys grown up. American Journal of Psychiatry, 155, 493-498.

Munk-Jorgensen, P. \& Mortensen, P. B. (1997) The Danish Psychiatric Central Register. Danish Medical Bulletin, 44, 82-84.

Rasmussen, P. \& Gillberg, C. (2000) Natural outcome of ADHD with developmental coordination disorder at age 22 years: a controlled, longitudinal, communitybased study. Journal of the American Academy of Child and Adolescent Psychiatry, 39, 1424-1431.

Seidman, L. J., Biederman, J., Faraone, S. V., et a (1997) A pilot study of neuropsychological function in girls with ADHD. Journal of the American Academy of Child and Adolescent Psychiatry, 36, 366-373.

Shaffer, D., Gould, M. S., Brasic, J., et al (1983) A children's global assessment scale (CGAS). Archives of General Psychiatry, 40, 1228-1231.

\section{Spencer, T., Biederman, J. \& Wilens, T. (1999)}

Attention-deficit/hyperactivity disorder and comorbidity. Pediatric Clinics of North America, 46, $915-927$.
SPSS Inc. (1998) SPSS for Windows, Release 9.0.0 Standard Version. Chicago, IL: SPSS Inc.

StataCorp (1999) Stata Statistical Software: Release 6.0. College Station, TX: Stata Corporation.

Weiss, G., Hechtman, L., Milroy, T., et al (1985) Psychiatric status of hyperactives as adults: a controlled prospective 15-year follow-up of 63 hyperactive children. Journal of the American Academy of Child Psychiatry, 24, 211-220

World Health Organization (1967) Internationa Classification of Diseases: Manual of the Internationa Statistical Classification of Diseases, Injuries and Causes of Death (ICD-8). Geneva: WHO

- (1992) The ICD-10 Classification of Mental and Behavioural Disorders: Clinical Descriptions and Diagnostic Guidelines. Geneva: WHO.

— (1999) World Health Organization, Assessment Classification and Epidemiology. http://www. who.int/ $\mathrm{msa} / \mathrm{mnh} / \mathrm{ems} / \mathrm{icdlO} /$ convtabl/intro.htm. 